\title{
Comportamento Adjunto: Controvérsias e Contribuições Teóricas ${ }^{1}$
}

\author{
Erica Maria Machado Santarém ${ }^{2}$ \\ Universidade São Francisco \\ Maria Teresa Araújo Silva \\ Universidade de São Paulo
}

\begin{abstract}
RESUMO - A área de investigação sobre comportamento adjunto, cujas pesquisas tiveram início na década de 60, é extensa e controvertida. O presente trabalho analisa as interpretações mais importantes do fenômeno, e mostra que diversas características apresentadas como próprias do comportamento adjunto também estão presentes em outros comportamentos. Falk interpretou o comportamento adjunto como atividade deslocada, Staddon como comportamento induzido por esquema, Timberlake como parte de um sistema comportamental pré-programado, e Wetherington questionou a existência de uma terceira classe de comportamento. Conclui-se que as tentativas de sistematização têm se mostrado incompletas devido à diversidade de topografias de comportamentos adjuntos e a sua dependência de variáveis tanto do esquema como da espécie e do ambiente.
\end{abstract}

Palavras-chave: comportamento adjunto; polidipsia; comportamento induzido por esquema; estímulo não contingente.

\section{Adjunctive Behavior: Controversial Issues and Theoretical Contributions}

\begin{abstract}
Dated from the sixties, the research field of adjunctive behavior is extensive and controversial. This review presents an analysis of the main theoretical interpretations of the phenomenon, and demonstrates that many of the features considered to be characteristic of adjunctive behavior are also present in non adjunctive behavior. Falk has interpreted adjunctive behavior as displaced activity, Staddon as schedule-induced behavior, Timberlake as a part of a pre-programmed behavioral system, and Wetherington has called into question the existence of a third class of behavior. It is concluded that all theoretical approaches appear to be incomplete due to the diversity of adjunctive behavior topographies and to their dependence on species and environmental variables, besides schedule parameters.
\end{abstract}

Key words: adjunctive behavior; polydipsia; schedule-induced behavior; non contingent stimulus.

A área de pesquisa sobre comportamento adjunto é extensa e controvertida. Diante da diversidade de comportamentos adjuntos observados sob esquemas contingentes e não contingentes, surgiram diferentes interpretações teóricas. O objetivo deste artigo é apresentar as mais importantes contribuições teóricas e as principais controvérsias ainda hoje não resolvidas na área. Após breve histórico, serão revistas as principais características do comportamento adjunto, destacando-se seu aspecto consensual ou controverso. A seguir serão expostas e analisadas as posições de Falk, Staddon, Timberlake e a crítica de Wetherington.

\section{Breve Histórico}

Em 1961, condicionando respostas de pressão à barra em sujeitos ratas mantidas sob privação alimentar (70\% a $80 \%$ do peso ad libitum), Falk verificou que os animais foram capazes de ingerir grandes quantidades de líquido de uma garrafa com água que ficara permanentemente disponí-

1 Apoio financeiro: FAPESP, CAPES.

2 Endereço: Universidade São Francisco, Faculdade de Educação e Ciências Sociais. Rua Hannemann, 352, Pari. CEP: 03031-040 São Paulo - SP. E-mail: esantarem@originet.com.br vel durante as sessões de condicionamento. O esquema de reforço utilizado para o condicionamento da resposta de pressão à barra foi um esquema de intervalos variáveis em que, a cada 60 segundos, em média, era liberada uma pelota de ração de $45 \mathrm{mg}$. A quantidade média de líquido ingerida pelos sujeitos do estudo de Falk, que não estavam privados de água, foi de $92,51 \mathrm{ml}$ por sessão de 3,17 horas de duração. Essa quantidade foi considerada excessiva pelo autor, desde que o nível médio de ingestão de água, pelos mesmos sujeitos, em sessões pré-experimentais, foi de $26,96 \mathrm{ml}$. Portanto, durante as sessões de condicionamento, os sujeitos aumentaram 3,43 vezes o consumo de água em relação ao nível pré-experimental de 24 horas medido nas gaiolas viveiros sob condição de alimentação livre. Esse fenômeno chamou a atenção de Falk sugerindo-lhe tratar-se de uma polidipsia (beber em excesso) de origem não fisiológica, porém comportamental, em ratos. Estudos posteriores levaram Falk (1969) a descartar definitivamente a hipótese de polidipsia fisiológica, ao mesmo tempo que outros comportamentos foram estudados, como por exemplo: agredir em pombos, lamber jatos de ar em ratos, correr em uma roda de atividade em ratos, roer madeira em ratos, ingerir álcool e outras drogas em ratos e macacos, defecar induzido em ratos, locomover-se em ratos e pombos, e o comportamento comer induzido em ratos (para uma revisão, ver Falk, 1971;Gimenes, 1988;Haydu, 
1988; Staddon, 1977 e Wetherington, 1982). Para uma revisão sobre ingestão de droga e comportamento adjunto, ver Sanger, 1986 e sobre polidipsia e alcoolismo, ver Tang \& Falk, 1988). Comportamentos adjuntos têm sido relatados também em seres humanos: ingestão de líquidos e atividade motora fina e global em adultos e crianças, agressão em crianças, fumar cigarros (para uma revisão, ver Haydu, 1994). Mais recentemente, a polidipsia em ratos vem sendo proposta como possível modelo animal de comportamento compulsivo em seres humanos (Santarem \& Silva, 1993; Woods \& Cols., 1993).

\section{Características controvertidas do comportamento adjunto}

Comparando polidipsia e outros comportamentos (agredir, lamber jatos de ar, correr e "pica"), Falk (1971) observou que os mesmos compartilhavam certas propriedades dinâmicas e eram função das mesmas variáveis. Descreveu algumas características que definiram o comportamento adjunto como uma classe especial de comportamento que se mostrava sob controle das mesmas variáveis e tinha os mesmos resultados comportamentais. Este argumento, no entanto, foi criticado posteriormente por Wetherington (1982), que ressaltou que muitas das características descritas como sendo exclusivas do comportamento adjunto podiam ser compartilhadas por comportamentos ou operantes ou respondentes; algumas delas, além disso, não se aplicavam adequadamente a comportamentos descritos como adjuntos. Segue-se a descrição de Falk e alguns dos contra-argumentos de Wetherington:

(a) Adjuntos são Comportamentos Pós-Alimento. Diversos comportamentos além da polidipsia foram descritos por Falk $(1971 ; 1977)$ como ocorrendo tipicamente no período pós-alimento, como por exemplo agredir em pombos, morder e pica em macacos e lamber jatos de ar em ratos. Agredir em pombos ocorreu tipicamente após o alimento no estudo de Campagnoni, Lawler e Cohen (1986). No entanto, certos casos que pareceram exceções para Falk (o agredir em pombos não foi pós-alimento quando o esquema utilizado foi um FR 60 e FR 120 , bem como a polidipsia ocorreu ao longo do intervalo com esquema de intervalo longo acima de $3 \mathrm{minu}-$ tos), para Wetherington (1982) foram considerados mais genéricos, porque um número crescente de estudos começaram a relatar comportamentos adjuntos que não ocorrem imediatamente após o alimento. Examinando esses estudos, a autora citou a polidipsia, em que o tempo entre a liberação do alimento e a primeira resposta de lamber o bebedouro foi uma função direta da duração do intervalo do esquema; a ingestão de uma solução de sacarina, que ocorreu, tanto após, quanto durante o intervalo entre liberações de alimento; o lamber um jato de ar, que ocorreu durante todo o intervalo, cessando apenas alguns segundos antes da liberação de alimento; e o correr na roda de atividade, que tem sido observado como um evento típico de meio de intervalo mais que de início do intervalo entre liberações de alimento. (b) O Comportamento Adjunto é Excessivo. Comportamentos adjuntos têm sido descritos como "excessivos" em relação à linha de base. É a característica que chamou a atenção de Falk, pela primeira vez, no estudo com polidipsia (Falk, 1961) e que ainda hoje se mantém como a mais importante. No entanto, é a característica mais controvertida quando se discute a adequação da linha de base e das medidas utilizadas para se definir o que é comportamento excessivo (Cohen \& Looney, 1984 Haydu \& Silva, 1997; Roper, 1981; Timberlake, 1982; Wetherington \& Brownstein, 1982; Wetherington, 1982). Apesar da controvérsia, o critério mais utilizado é o de Roper, segundo o qual um comportamento é excessivo se sua taxa geral de ocorrência sob um esquema intermitente excede sua taxa de ocorrência sob (1) uma condição de linha de base maciça em que a mesma quantidade de alimento é toda disponível no início da sessão, e (2) uma condição de "extinção" em que nenhuma quantidade de alimento é apresentada ao sujeito nas sessões de linha de base. Quanto ao tipo de medida, Staddon (1977) comentou que as medidas mais apropriadas seriam: taxa de ingestão $(\mathrm{ml} / \mathrm{m})$, taxa de beber (lambidas/ $\mathrm{m})$, ou a medida de duração do comportamento de beber.

(c) O Adjunto é uma Função em "U" Invertido. A duração do intervalo entre as liberações de estímulo tem sido referida como um dos principais determinantes do comportamento adjunto. Falk (1966b) foi o primeiro a investigar essa variável e mostrou uma relação funcional em forma de $U$ invertido entre o grau de polidipsia e a duração do intervalo. A partir de durações entre 4 e $5 \mathrm{~s}$, a função é crescente, atingindo um pico de intensidade máxima entre intervalos de 120 a 180 s, aproximadamente. A função é gradativamente decrescente para intervalos de durações maiores. A medida utilizada por Falk foi volume de água ingerida por sessão e, posteriormente, outros estudos com medidas diferentes também mostraram a função em $U$ invertido: número de respostas de lamber por sessão (Burks, 1970; Flory, 1971; Wetherington, 1979); duração de beber por pelota (Wetherington, 1979) e porcentagem de ocorrência de beber por pelota (Allen \& Kenshalo, 1976; Segai, Oden \& Deadwyler, 1965; Wetherington, 1979). Por outro lado, medidas de volume de água ingerida e número de respostas de lamber por unidade de tempo, e a medida de porcentagem de tempo dispendido com beber foram todas uma função decrescente da duração do intervalo (para revisão, ver Wetherington, 1979). Porém, Roper (1980) obteve a função em $U$ invertido para a medida de taxa de beber (vol/m). Wetherington (1982), fazendo um paralelo entre a polidipsia e a resposta eliciada por um estímulo incondicionado, examinou vários estudos mostrando a mesma função em $U$ invertido entre a resposta incondicionada e o intervalo entre apresentações do estimulo incondicionado.

A função bitônica que caracteriza a polidipsia foi observada com ataque em sujeitos pombos (Flory, 1969); fuga do esquema em pombos (Brown \& Flory, 1972); com o com- 
portamento de morder em sujeitos macacos submetidos a esquema de FI de liberação de alimento ou choque (Deweese, 1977) e com o comportamento de roer em ratos submetidos a esquema FI de liberação de comida (Roper, 1980). Foi obtida de modo semelhante em crianças para os comportamentos de beber, de "movimentos motores finos" e de "limpeza" \{ grooming) (Granger, Porter \& Christoph, 1984).

(d) O Comportamento Adjunto Pode Manter um Operante. A polidipsia ocorre mesmo quando se exige que o animal pressione uma barra em um determinado número de vezes (esquema FR) para ter acesso a uma garrafa de água. Falk (1966a) tomou essa característica da polidipsia como indicador de que o comportamento adjunto tem propriedades reforçadoras ou "motivacionais", propriedades também demonstradas com o comportamento de agredir induzido pela extinção. No entanto, Wetherington (1982) comentou que essa característica não é exclusiva do comportamento adjunto e que diversos comportamentos, como por exemplo comer, beber, agredir e copular, eliciados por estimulação elétrica do cérebro, podem manter um comportamento operante.

(e) O Comportamento Adjunto é uma Função Inversa do Peso Corporal. A polidipsia foi mantida em um mesmo nível quando o grau de privação dos sujeitos variou entre 80 e $90 \%$ do peso ad libitum. Porém, quando o peso corporal dos sujeitos variou acima de $95 \%$ desse peso, a polidipsia mostrou-se acentuadamente decrescente (Falk, 1969). A mesma relação foi obtida para o comportamento de lamber jatos de ar (Chillag \& Mendelson, 1971) e agredir induzido pelo esquema (citado por Wetherington, 1982). Wetherington comentou que a privação de alimento facilita a ocorrência de vários comportamentos, como por exemplo o correr em uma roda de atividade, o cavar areia, a pressão à barra não reforçada, a agressão eliciada por choque, etc. A autora sugeriu que a liberação intermitente de alimento serve para "sensibilizar" certos reflexos e a propriedade de sensibilização da intermitência do alimento é "sensibilizada" pela privação. Essa interpretação resolveria o aparente paradoxo de privação de alimento resultar em diminuição do consumo de água, quando a privação de alimento intensifica a polidipsia. Para Wetherington, a operação que produz polidipsia não é privação de alimento, mas a intermitência do alimento. Assim como, a ingestão de água em ratos privados aumenta quando estes são alimentados. Do mesmo modo, no procedimento de polidipsia, a ingestão de água aumenta quando o alimento é liberado e ingerido pelos sujeitos. Recentemente, Todd, Cunningham, Janes, Mendelson \& Morris (1997) conseguiram desenvolver e manter polidipsia em ratos sem redução de peso, apoiando a interpretação de Wetherington da polidipisa induzida como um responder eliciado e sensibilizado pela apresentação repetida do alimento.

(f) O Comportamento Adjunto é Afetado pelo Ambiente. Falk (1971) referiu-se a alguns estudos que mostraram que a polidipsia é atenuada quando outros estímulos, além da garrafa de água, estão presentes na situação, como por exemplo uma roda de atividade, papel colocado sob a grade do piso da caixa experimental, qualquer material possível de roer, etc. Wetherington (1982) comentou que essa característica do comportamento adjunto é compartilhada, tanto pelo comportamento operante, como pelo comportamento respondente, ou seja, ambos são também afetados por circunstâncias ambientais.

(g) Não é Necessário um Esquema Contingente. Embora a polidipsia tenha sido observada primeiro durante o condicionamento da resposta operante de pressão à barra sob um esquema VI (Falk, 1961), posteriormente foi observada também com esquemas de liberação não contingente de alimento (Falk, 1966a; Schurtleff, Delamater \& Riley, 1983). Outros comportamentos adjuntos, como agredir, lamber jatos de ar e "pica", foram observados sob esquemas não contingentes (FT) (citados por Falk, 1971). Para Wetherington, isso é evidência de que o comportamento adjunto não deve ser interpretado como um sub-produto ou efeito colateral do esquema de reforço, mas como um resultado direto da intermitência do estímulo.

\section{Contribuições Teóricas e Controvérsias Principais}

(a) Falk: Comportamento Adjunto como Atividade Deslocada. Falk em 1977 e, posteriormente, em 1986, desenvolveu o conceito de comportamento adjunto como atividade deslocada. Situações em que ocorrem comportamentos adjuntos são semelhantes a situações em que ocorrem atividades deslocadas. Resumidamente, as chamadas atividades deslocadas tendem a ocorrer em situações de conflito em que respostas incompatíveis são simultaneamente ativadas, como por exemplo, ataque versus fuga, sexo versus ataque, ou em situações de impedimento, por bloqueio físico de uma resposta parcialmente ativada ou pela presença de predador. Da mesma maneira, operações de esquemas ou de liberação intermitente de alimento definem uma situação de bloqueio de um comportamento (comer) altamente ativado pela operação de privação. Falk (1977) ressaltou que estudos etológicos têm reconhecido que o comportamento deslocado é também função não apenas das situações de conflito ou de bloqueio do comportamento consumatório, mas também dos estímulos ambientais presentes na situação de conflito. Nesse sentido, portanto, haveria similaridade nos processos determinando o deslocamento e o comportamento adjunto. Ambos ocorreriam quando um comportamento altamente ativado é impedido; e ambos, comportamento deslocado e adjunto, são função dos estímulos presentes no ambiente. Embora aparentemente fora de contexto, ambos os comportamentos compartilhariam uma função adaptativa para o organismo.

A função adaptativa do comportamento adjunto ou do comportamento deslocado seria a de manter o sujeito na situação de conflito quando esta é altamente favorável e o comportamento de fuga ineficaz (Falk, 1977; 1986). Por 
exemplo, quando a taxa de liberação de alimento de um esquema é baixa, a probabilidade do comportamento de aproximação (dirigido ao comedouro) é também baixa e a do comportamento de fuga (afastamento do comedouro) é alta. Ao contrário, quando a taxa de liberação de alimento é alta, a probabilidade do comportamento de aproximação é alta e a do comportamento de fuga é baixa. O comportamento adjunto ocorre na faixa de conflito, sendo de intensidade máxima quando a taxa de liberação de alimento gera uma probabilidade igual de comportamento de aproximação e de fuga. Ele é adaptativo no sentido de que é uma resposta adequada do organismo a uma situação de conflito passageiro, pois evita que o animal abandone a fonte de alimento.

Porém, essa explicação aparentemente não se aplicaria ao comportamento adjunto em ambiente de laboratório. Falk (1986) defendeu-se dessa objeção argumentando que a função adaptativa do comportamento adjunto seria, não só de origem ontogenética, como também filogenética e, por isso, comportamentos adjuntos ocorreriam mesmo sem uma função adaptativa explícita como é o caso em um ambiente experimental de laboratório.

Ao modelo de comportamento adjunto como atividade deslocada se conformaria, segundo Falk (1986), o uso abusivo de drogas, bem como a formação e função de comportamentos atualizados em seres humanos. Com relação ao uso abusivo de drogas, o comportamento adjunto não seria adaptativo na medida em que as conseqüências são danosas para o indivíduo. Segundo o autor, o caráter nocivo ou adaptativo do comportamento adjunto é função não só das contingências do esquema, mas também dos outros componentes do contexto ambiental.

O conceito de Falk sobre comportamento adjunto extrapola o ambiente de laboratório e procura explicar comportamentos humanos complexos, como comportamento de rituais, tabus e dependência de droga. Na medida em que propõe maior generalidade do fenômeno, distancia-se das questões controvertidas que ainda existem sobre a generalização (Diosdado, 1984;Haydu, 1988;Roper, 1981)ecaracterização do comportamento adjunto como uma terceira classe de comportamento (Wetherington, 1982).

(b) Staddon: Comportamento Induzido pelo Esquema. Para Staddon (1977), o comportamento adjunto é um exemplo de comportamento induzido pelo esquema. Desde sua colocação, introduziu-se o uso dos termos "adjunto" e "induzido por esquema" como sinônimos na literatura corrente.

Staddon não foi o primeiro a se referir ao comportamento adjunto como induzido pelo esquema. Segai (1972) referiu-se ao esquema de liberação intermitente de alimento como uma operação de indução emocional para diferenciá-lo de outras operações de indução, tais como indução por reforço (modelagem), indução por privação, indução por eliciação reflexa e indução por estímulos eliciadores de padrões instintivos. A característica comum entre todas essas operações é propiciar ou permitir o aparecimento de topografias de respostas sujeitas à ação da contingência. A operação de indução emocional envolveria a apresentação espaçada e repetida de algum estímulo, como por exemplo alimento, choque, etc. Também são operações de indução emocional a extinção, a apresentação pareada de um estímulo neutro e um estímulo aversivo sobre uma linha de base operante (procedimento CER), os esquemas múltiplos e a remoção de um estímulo estampado.

Staddon (1977) classificou os comportamentos sob esquema em três categorias diferentes, segundo a distribuição no intervalo entre as apresentações do alimento. Estudos anteriores de observação do comportamento de pombos (Staddon \& Simmelhag, 1971) e de ratos (Staddon \& Ayres, 1975) haviam mostrado que os sujeitos desenvolviam uma seqüência regular de comportamento durante a liberação periódica de alimento. Essa seqüência caracterizava-se por alguns comportamentos que ocorriam tipicamente no início do intervalo (caminhar em passos, dar voltas em círculo e bater as asas para pombos; beber água e correr para ratos quando uma garrafa de água e uma roda de atividade estavam disponíveis), e por outros comportamentos que aumentavam de frequiência no segmento terminal do intervalo pós-alimento (bicar para pombos; roer, ou "cavar" o comedouro para ratos). Os comportamentos que ocorriam na porção inicial e terminal entre a liberação do alimento foram denominados por Staddon e Simmelhag (1971) de, respectivamente, comportamento interino e resposta terminal. O comportamento terminal é tipicamente estereotipado, freqüentemente semelhante na forma à resposta consumatória; aumenta de freqüência com o passar do tempo e usualmente ocorre ao final do intervalo quando o alimento fica disponível. O comportamento interino ocorre ao início do intervalo quando a probabilidade de alimento é baixa e, geralmente, é incompatível com a resposta terminal. A resposta terminal e o comportamento interino correspondem àqueles comportamentos que na terminologia de Staddon (1977) são "facilitados" ( por comparação com uma linha de base pré e pós esquema quando nenhum estímulo, alimento, é liberado) ou induzidos pelo esquema. Staddon também identificou uma terceira classe de comportamento, o comportamento facultativo, que não é induzido ou facilitado pelo esquema. Comportamentos dessa classe ocorreriam, geralmente, na porção média do intervalo quando a tendência para o sujeito engajar-se nas duas classes dominantes - interina e terminal - é fraca. "Correr na roda de atividade" seria um exemplo de comportamento facultativo. No estudo de Staddon e Ayres (1975), o correr na roda de atividade surgiu, em maior intensidade, na porção média do intervalo entre liberações de alimento e em seguida ao comportamento de beber água. Quando a liberação intermitente de alimento foi interrompida, o tempo dispendido em correr aumentou, inicialmente, e manteve-se estável em níveis altos, enquanto beber e engajarse em outras atividades diminuíram. Esses dados, portanto, sugeriram a Staddon e Ayres e Staddon (1977) que o comportamento de correr, embora presente na seqüência comportamental, não era função da liberação intermitente de alimento, tal como beber (comportamento interino) e "antecipar" o alimento (comportamento terminal).

Não se deteve Staddon apenas em descrever e classificar o comportamento, mas também propôs uma hipótese 
motivacional para explicar os comportamentos observados nos esquemas de liberação periódica de alimento. Em 1977 propôs a hipótese da indução de dois estados motivacionais antagônicos e, posteriormente, substituiu-a pela hipótese de um único estado motivacional relacionado ao estímulo consumatório e predominante na situação de esquema. De acordo com a hipótese de dois estados motivacionais antagônicos, os comportamentos interinos e a resposta terminal corresponderiam, cada um deles, a um determinado estado motivacional. Na porção final do intervalo, quando a liberação do alimento é iminente, o estado motivacional predominante seria aquele relacionado à obtenção do alimento. $\mathrm{Na}$ porção inicial do intervalo, quando a probabilidade de alimento é baixa, o estado motivacional predominante e seu comportamento correspondente seria um estado antagônico ao do período terminal do intervalo; como exemplo, Staddon se referiu à sede induzida no caso da polidipsia. Os comportamentos interinos que ocorressem no período inicial do intervalo dependeriam dos estímulos ambientais disponíveis (por exemplo, se houvesse água disponível, o sujeito beberia). Conforme o tempo evolui da porção inicial do intervalo para a porção final, o estado motivacional antagônico e seus comportamentos associados começariam a se dissipar, e um estado motivacional relacionado à proximidade do alimento e à resposta terminal gradualmente aumentaria até a liberação do alimento. Se o intervalo entre liberações de alimento for muito curto (Staddon \& Simmelhag, 1971), observa-se apenas comportamento interino e comportamento terminal, havendo um período no meio do intervalo quando os dois comportamentos deverão estar em conflito direto. Por outro lado, se o período entre a liberação do alimento for bastante longo (Staddon \& Ayres, 1975) nenhum dos dois estados motivacionais referidos deverão dominar no meio de intervalo e, assim, o comportamento facultativo terá oportunidade de se manifestar.

A proposta de Staddon sobre a indução de dois estados motivacionais antagônicos e seus comportamentos correspondentes não se confirma em estudos posteriores com liberação de alimento a intervalos longos e com liberação de água ao invés de alimento, realizados por ele próprio e colaboradores (Innis, Reberg, Mann, Jacobson \& Turton, 1983; Innis, Simmelhag-Grant \& Staddon, 1983; Reberg, Innis, Mann \& Eizenga, 1978). De modo geral, os resultados desses estudos mostraram que a resposta terminal de bicar, observada em pombos com esquemas de intervalo de curta duração entre a liberação de alimento ou água, não é observada com esquemas de intervalo de longa duração. O comportamento interino durante esquemas de liberação de água foi, principalmente, ficar parado com a cabeça no bebedouro e quando ocorria alguma movimentação, era ir e voltar rapidamente do fundo da caixa para o bebedouro, independente da duração do intervalo (de 10 a 30s). Por outro lado, em esquemas de liberação de alimento, o comportamento interino apresentou maior variabilidade à medida que aumentou a duração do intervalo, e a distinção entre comportamento interino e comportamento terminal não foi tão característica como em intervalos de curta duração.
A partir das constatações acima, a hipótese de indução de dois estados motivacionais antagônicos foi substituída pela hipótese de um único estado motivacional relacionado ao estímulo e predominante na situação de esquema. Innis, Simmelhag-Grant e Staddon (1983) afirmaram que o comportamento dos sujeitos, durante esquemas de intervalos curtos, seria facilmente explicável pela hipótese de dois estados motivacionais antagônicos, mas o comportamento, durante intervalos mais longos, não era explicado. Os comportamentos durante intervalos, curtos ou longos, entre liberações de estímulo poderiam ser mais adequadamente explicados pela hipótese de um único estado motivacional relacionado ao alimento ou à água, ao invés de dois estados. A resposta terminal, ao final do intervalo, e o comportamento interino, no início do intervalo, seriam ambos funções de um mesmo estado motivacional predominante ao longo do intervalo de tempo definido pelo esquema.

(c) Timberlake: Sistema de Comportamento. Timberlake e Lucas (1985), em um extenso estudo sobre a organização comportamental subjacente ao comportamento supersticioso em pombos, concluíram que a organização do comportamento é mais complexa e flexível do que sugere a organização em interino e terminal. Os autores observaram que o comportamento interino de pombos, afastando-se e retornando em seguida ao comedouro após a ingestão de alimento, parece ser um padrão de busca pós-alimento típico da espécie, ajustado a situações de laboratório em que o alimento reaparece periodicamente no mesmo local. Quando o comedouro foi fixado ao piso, no centro da caixa experimental, simulando o ambiente natural da espécie, os autores observaram que o comportamento de mover-se em círculo na área de alimento predominou na maioria dos sujeitos. Para Timberlake e Lucas, a organização temporal do comportamento sob esquema, mais do que corresponder a estados motivacionais diferentes, parece representar um padrão típico da espécie relacionado ao comportamento de busca e ingestão de alimento. Os dados com pombos, no estudo de Reberg, Innis, Mann e Eizenga (1978), em que o comportamento de comer não foi induzido pela liberação de água mostrando que o comportamento predominante é permanecer na área de água e não afastar-se, são portanto compatíveis com a presente interpretação. Segundo Timberlake e Lucas, o comportamento de permanecer na área de liberação de água é de se esperar, pois no ambiente natural as fontes de água encontram-se mais freqüentemente concentradas e não espalhadas em pequenas porções, como é o caso do alimento. Pondo à prova empiricamente essa interpretação, Lucas, Timberlake e Gawley (1988) estudaram sujeitos ratos submetidos à liberação regular de alimento durante 24 horas. Os autores manipularam intervalos crescentes de tempo (FT 16 aFT512 segundos), e diversas categorias comportamentais foram observadas em níveis percentuais mais elevados durante os esquemas de FT do que durante a condição de linha de base de 24 horas de alimento irrestrito. Paralelamente ao efeito 
de intensificação, os autores puderam constatar que a organização do comportamento nas pausas impostas pelo esquema FT não diferiu da organização do comportamento nas pausas ocorridas durante a ingestão de alimento irrestrito na linha de base. Lucas e colaboradores sugeriram que o comportamento sob esquema de alimento pareceu consistir, mais uma vez, de um conjunto de respostas complexas funcionalmente relacionadas ao comportamento de forragear. Em 1991, Timberlake e Lucas estudaram o comportamento sob liberação intermitente de água, confirmando que a ausência de indução do comer nessa situação estaria relacionada ao conjunto de respostas ligadas ao comportamento de busca de água no ambiente natural. Resumindo, Timberlake e Lucas (1985; 1991) e Lucas, Timberlake e Gawley (1988) argumentaram que o comportamento adjunto que aparece sob esquemas de liberação regular de alimento não é um comportamento estranho, mas uma distribuição ordenada de respostas comumente relacionadas ao comportamento de comer e forragear em animais. Os autores sugerem a existência de sistemas complexos de comportamento pré-programados filogeneticamente e organizados em torno de eventos biológicos relevantes, tal como o alimento.

A posição de Timberlake e colaboradores, acima descrita, tem implicações para o problema da generalidade do comportamento adjunto. Sendo o comportamento organizado em torno de eventos biológicos relevantes, espera-se que a ocorrência do comportamento adjunto sofra restrições quanto à espécie envolvida e à natureza do estímulo consumatório. Alguns estudos mostraram restrições quanto à natureza do estímulo consumatório (Haydu, 1988; Haydu, Luzia, Marinho, Maciel \& Silva, 1991; Wetherington \& Brownstein, 1977; Wetherington \& Riley, 1985); outros têm mostrado restrições relativas à espécie, isto é, o comportamento adjunto observado em uma determinada espécie não é observado em outra (Anderson \& Shettleworth, 1977; Shearon \& Allen, 1989; Wilson \& Spencer, 1975). Esses estudos mostram, é certo, que a organização temporal do comportamento diferencia-se de espécie para espécie e depende da natureza do estímulo consumatório. Porém, permanece controvertida a questão: o comportamento adjunto resulta de uma relação de contingência entre resposta e estímulo conseqüente?

(d) A Crítica de Wetherington. Em uma revisão crítica e ampla da área de comportamento adjunto, Wetherington (1982) questiona a existência de uma terceira classe de comportamento além das categorias respondente e operante já descritas (Skinner, 1938). A autora mostra que o comportamento adjunto pode apresentar características de operante, bem como características de respondente, muito embora classificações em uma ou outra área sejam muitas vezes prematuras e inadequadas. Enquanto operante, existem demonstrações convincentes de controle do comportamento adjunto (polidipsia) por variáveis que afetam o comportamento operante, tais como qualidade e quantidade do alimento e privação. Além disso, verificam-se com a polidipsia os fenô- menos de contraste e generalização de estímulo, e a equação de Herrnstein (1970) descreve a relação entre a taxa de beber induzido pelo esquema e a taxa de liberação de alimento. Por outro lado, não há provas seguras de que a polidipsia seja afetada por estímulos conseqüentes. Enquanto respondente, Wetherington examinou as principais objeções que têm sido levantadas à visão de comportamento adjunto como eliciado, e concluiu que são inadequadas. Paralelamente, demonstrou algumas correspondências entre comportamento respondente e comportamento adjunto: a polidipsia mostrou-se classicamente condicionável, e a relação existente entre comportamento adjunto e duração do intervalo entre alimento é semelhante à relação entre o intervalo de apresentação do estímulo incondicionado (US) e a resposta incondicionada (UR) em estudos envolvendo a manipulação do intervalo entre apresentações de US; o aumento de intensidade do US tem um efeito sobre a UR que é semelhante ao da quantidade de alimento sobre o comportamento adjunto. Em ambos os casos, a função parece ser crescente, segundo a autora, embora existam relatos de uma função bitônica.

A partir das constatações acima, Wetherington (1982) posicionou-se contra tentativas de classificação do comportamento adjunto quer como uma nova categoria, quer como incluso em uma das categorias tradicionais (operante e respondente). Sugeriu que, antes de qualquer classificação, os estudos se voltem para investigações sobre os tipos de comportamento que ocorrem em situações de estímulos não contingentes em função das variáveis manipuladas, tais como: características do estímulo (intensidade, duração, qualidade, intervalo de apresentação, etc); natureza da estimulação interna e externa presentes na situação, e natureza dos objetos em direção aos quais o comportamento pode ser dirigido.

\section{Comentários Finais}

O objetivo dessa revisão foi mostrar o quanto é extensa e controvertida a área de investigação sobre comportamento adjunto e o quanto tentativas de sistematização têm se mostrado incompletas. Em parte isso se deve à diversidade de comportamentos que ocorrem sob esquemas contingentes e não contingentes e à dependência desses comportamentos de variáveis tanto do esquema como da espécie e do ambiente sobre o qual atua o organismo. Parece existir unanimidade com relação à organização temporal do comportamento sob esquema, ou seja, o comportamento no intervalo se distribui em função da maior ou menor proximidade da liberação do estímulo consumatório. Wetherington e Brownstein (1977) sugeriram o termo modulado pelo esquema para se referir ao controle temporal do esquema sobre o comportamento induzido e não induzido. Contudo, o termo não esclarece a questão da intensificação do comportamento sob esquema, que não seja explicada pela associação direta do comportamento com o estímulo consumatório. Parece que, na indução, a natureza do estímulo é uma variável tão importante quanto 
a interrnitência. Comportamentos são mais dificilmente induzidos quando o estímulo consumatório é água, sugerindo que a indução seja um fenômeno restrito ao alimento. As teorias apresentadas contribuem parcialmente para a explicação do fenômeno na medida em que abordam alguns de seus aspectos, mas não todos. A classificação do comportamento sob esquema em categorias distintas (interinos, facultativos e terminais) é pouco elucidativa na medida em que essas categorias não são replicáveis em outras espécies e dependem da natureza do estímulo. A organização do comportamento em torno de eventos biologicamente relevantes explica a topografia dos comportamentos observados, mas é insuficiente para explicar por que alguns comportamentos são intensificados e outros não. O comportamento induzido como atividade deslocada parece não resistir ao teste experimental no laboratório (Roper \& Posadas-Andrews, 1981).

$\mathrm{Na}$ última década, as publicações sobre comportamento adjunto têm se concentrado sobre a polidipsia. Dentre os estudos com polidipsia, muito tem-se investigado sobre o efeito da manipulação de drogas ou lesões neuroquímicas com vistas a entender o controle central desse comportamento, como por exemplo, os estudos de Bowers, Halberda, Mullen e May (1997); Cirulli, Van Oers, De Kloet e Levine (1994); Didriksen, Olsen, e Christensen (1993); Flores e Pellon (1997); Lu, Tseng, Wan, Yin e Tung (1992); Mittleman, Blaha, e Phillips (1992). Os estudos sem droga manipulam variáveis diversas, porém a mais consistentemente investigada tem sido a consequiênciação da polidipsia em desenvolvimento ou já instalada (Allan \& Matthews, 1992; Lamas \& Pellon, 1995a, 1995b; Lamas \& Pelon, 1997). Esses estudos têm mostrado que o comportamento induzido é sensível ao controle operante. Embora a contingência não seja a variável crítica no desenvolvimento da polidipsia, ela parece, no entanto, modificar a polidipsia já instalada.

Publicações sobre outros comportamentos adjuntos contribuem para a discussão sobre a generalidade do fenômeno. Assim, demonstram a indução de comportamentos ainda não investigados, como por exemplo, lamber a pata em rato (Lawler \& Cohen, 1992), hábitos orais excessivos em humano (Gramling, Grayson, Sullivan \& Schwartz, 1997) e replicam em outras espécies os adjuntos já demonstrados no rato, como por exemplo, a defecação em pombos (Jarema, LeSage, \& Poling 1995).

Os estudos teórico-metodológicos dividem-se entre aqueles que, com base em problemas examinados, questionam a generalidade do fenômeno (Overskeid, 1992; Haydu \& Silva, 1997), e aqueles que reafirmam a adequação dos estudos como demonstrações válidas do comportamento adjunto em animais e humanos (Pellon, 1990; Falk, 1994; Falk, 1998; Falk \& Kupfer, 1998).

Diante das controvérsias teóricas e metodológicas, a proposta de Wetherington (1982) ainda se mantém como a mais parcimoniosa: deixar de lado qualquer classificação do comportamento e "mapear" relações funcionais entre o comportamento e os vários procedimentos de liberação não contingente de diferentes estímulos.

\section{Referências}

Allan, R.W. \& Matthews, T.J. (1992). Selective sensitivity of schedule-induced activity to an operant suppression contingency. Journal of the Experimental Analysis of Behavior, 58,471-483.

Allen, S.S. \& Kenshalo, D.R. (1976). Schedule induced drinking as a function of interreinforcement interval in the rhesus monkey. Journal of the Experimental Analysis of Behavior, 12, 825-828.

Anderson, M.C. \& Shettleworth, S.J. (1977). Behavioral adaptation to fixed-interval and fixed-time food delivery in golden hamsters. Journal of the Experimental Analysis of Behavior, 25, 33-49.

Bowers, R.L., Halberda, J., Mullen, L. \& May, K. (1997). Captopril alters schedule induced polydipsia, urination, and defecation in rats. Pharmacology, Biochemistiy \& Behavior, 57, 343-359.

Brown, T.G. \& Flory, R.K. (1972). Schedule-induced escape from fixed-interval reinforcement. Journal of the Experimental Analysis of Behavior, 17, 395-403.

Burks, CD. (1970). Schedule-induced polydipsia: Are responsedependent schedules a limiting condition? Journal of the Experimental Analysis of Behavior, 13, 351-358.

Campagnoni, F.R., Lawler, C.P \& Cohen, P.S. (1986). Temporal patterns of reinforcement-induced general activity and attack in pigeons. Physiology \& Behavior, 37, 577-582.

Chillag, D. \& Mendelson, J. (1971). Schedule-induced air licking as a function of body-weight deficit in rats. Physiology \& Behavior, 6, 603-605.

Cirulli, R, Van Oers, H., De Kloet, E.R. \& Levine, S. (1994). Differential influence of corticosterone and dexamethasone on schedule-induced polydipsia in adrenalectomized rats. Behavior Brain Research, 65, 33-39.

Cohen, P.S. \& Looney, T.A. (1984). Induction by reinforced schedule. Journal of the Experimental Analysis of Behavior, 41,345353.

Deweese, J.O. (1977). Schedule-induced biting under fixed-interval schedules of food or electric shock. Journal of the Experimental Analysis of Behavior; 27, 419-431.

Didriksen, M. Olsen, G.M. \& Christensen, A.V. (1993). Effect of clozapine upon schedule-induced polydispia (SIP) resembles neither the actions of dopamine D1 nor D2 blockade. Psychopharmacology, 113, 250-256.

Diosdado, E.T. (1984). A construção de ninho no hamster como comportamento induzido pelo esquema: a questão da generalidade dos efeitos da indução. Dissertação de Mestrado, Universidade de São Paulo, São Paulo.

Falk, J.L. (1961). Production of polydipsia in normal rats by an intermittent food schedule. Science, 133, 195-196.

Falk, J.L. (1966a). The motivational properties of schedule induced polydipsia. Journal of the Experimental Analysis of Behavior, 9, 19-36.

Falk, J.L. (1966b). Schedule induced polydipsia as a function of fixed interval lenght. Journal of de Experimental Analysis of Behavior, 9, 37-39.

Falk, J.L. (1969). Conditions producing psychogenic polidipsia in animals. Annals of the New York Academy Science, 157, 569593. 
Falk, J.L. (1971). The nature and determinants of adjunctive behavior. Physiology \& Behavior, 6, 577-588.

Falk, J.L. (1977). The origin and functions of adjunctive behavior. Animal Learning \& Behavior, 5, 325-335.

Falk, J.L. (1986). The formation and function of ritual behavior. Em T. Thompson \& M.D. Zeiler (Orgs.), Analysis and integration of behavioral units (pp. 335-355). Hillsdale, N.J.: Lawrence Erlbaum Associates, Inc.

Falk, J.L. (1994). Schedule-induced behavior occurs in humans: A reply to Overskeid. Psychological Record, 44, 45-62.

Falk, J.L. (1998). Drug abuse as adjunctive behavior. Drug and Alcohol Dependence. Psychological Record, 52, 91-98.

Falk, J.L. \& Kupfer, A.S. (1998). Adjunctive behavior: application to the analysis and treatment of behavior problems. Em O'Donohue, W.T. (Org.), Learning and behavior therapy (pp. 334-351). Boston, U.S.A.: Hardcover.

Flores, P. \& Pellon, R. (1997). Effects of d-amphetamine on temporal distributions of schedule-induced polydipsia. Pharmacology, Biochemistry \& Behavior, 57, 81-87.

Flory, R.K. (1969). Attack behavior as a function of minimum inter-food interval. Journal of the Experimental Analysis of Behavior, 12, 825-828.

Flory, R.K. (1971). The control of schedule-induced polydipsia frequency and magnitude of reinforcement. Learning \& Motivation, 2, 215-227.

Gimenes, L.S. (1988). Comportamento induzido por contingências [Resumo]. Em Sociedade Brasileira de Psicologia (Org.), Resumos de comunicações científicas, XVIII Reunião Anual de Psicologia (67-72). Ribeirão Preto: SBP.

Gramling, S.E., Grayson, R.L., Sullivan, T.N. \& Schwartz, S. (1997). Schedule-induced masseter EMG in facial pain subjects vs. no-pain controls. Physiology and Behavior, 61, 301309.

Granger, R.G., Porter, J.H. \& Christoph, N.L. (1984). Scheduleinduced behavior in children as function of interreinforcement interval length. Physiology \& Behavior, 33, 153-157.

Haydu, V.B. (1988). Limitações na indução do comportamento de roer madeira em ratos: um estudo com privação de água. Dissertação de Mestrado, Universidade de São Paulo, São Paulo.

Haydu, V.B. (1994). Indução de comportamentos por esquemas: um estudo com seres humanos. Tese de Doutorado, Universidade de São Paulo, São Paulo.

Haydu, V.B., Luzia, J.C., Marinho, M.L., Maciel, S.V. \& Silva, M.T.A. (1991). Indução de comportamento por esquema de liberação de água ou alimento: um estudo comparativo [Resumo]. Em Sociedade Brasileira de Psicologia (Org.), Resumos de comunicações científicas, XXI Reunião Anual de Psicologia (p. 98). Ribeirão Preto: SBP.

Haydu, V.B. \& Silva, M.T.A. (1997). Indução de comportamento por esquema: análise metodológica e conceituai. Acta Comportamentalia, 5, 31-57.

Herrnstein, RJ. (1970). On the law of effect. Jornal of the Experimental Analysis of Behavior, 13, 243-266.

Innis, N.K., Reberg, D., Mann, B., Jacobson, J. \& Turton, D. (1983). Schedule-induced behavior for food and water: effects of interval duration. Behavior Analysis Letters, 3, 121-200.
Innis, N.K., Simmelhag-Grant, V.L. \& Staddon, J.E.R. (1983). Behavior induced by periodic food delivery: the effects of interfood interval. Journal of the Experimental Analysis of Behavior, 39, 309-322.

Jarema, K., LeSage, M. \& Poling, A. (1995). Schedule-induced defecation: A demonstration in pigeons exposed to fixed-time schedules of food delivery. Physiology and Behavior, 58, 195198.

Lamas, E. \& Pellon, R. (1995a). Food-delay duration and the development of schedule-induced polydispia in rats. Physiology and Behavior, 57, 1221-1224.

Lamas, E. \& Pellon, R. (1995b). Food-deprivation effects on punished schedule-induced drinking in rats. Journal of the Experimental Analysis of Behavior, 64, 47-60.

Lamas, E. \& Pellon, R. (1997). Food deprivation and food-delay effects on the development of adjunctive drinking. Physiology and Behavior, 61, 153-158.

Lawler, C.R \& Cohen, P.S. (1992). Temporal patterns of scheduleinduced drinking and pawgrooming in rats exposed to periodic food. Animal Learning and Behavior, 20, 266-280.

Lu C.C., Tseng, C.J., Wan, F.J., Yin, T.H. \& Tung, C.S. (1992). Role of locus coeruleus and serotonergic actions on scheduleinduced polydipsia. Pharmacology, Biochemistry \& Behavior, $43,255-261$.

Lucas, G.A., Timberlake, W. \& Gawley, D.J. (1988). Adjunctive behavior of the rat under periodic food delivery in a 24-hour environment. Animal Learning \& Behavior, 16, 19-30.

Mittleman, G., Blaha, CD. \& Phillips, A.G. (1992). Pituitary-adrenal and dopaminergic modulation of schedule-induced polydipsia: Behavioral and neurochemical evidence. BehaviorNeuroscience, 106, 408-420

Overskeid, G. (1992). Is any human behavior schedule-induced? Psychological Record, 42, 323-340.

Pellon, R. (1990). Polidipsia inducida por programa: I. Definicion y marco conceptual. Revista de Psicologia Generaly Aplicada, $43,313-326$.

Reberg, D., Innis, N.K., Mann, B. \& Eizenga, C. (1978). "Superstitious" behavior resulting from periodic response-independent presentations of food or water. Animal Behavior, 26, 507519.

Roper, T.J. (1980). Changes in rate of schedule induced behavior in rats as a functionof fixed-interval schedule. Quaterly Journal of Experimental Psychology, 32, 159-170.

Roper, T.J. (1981). What is meant by the term "schedule-induced" and how general is schedule induction? Animal Learning \& Behavior, 9, 433-440.

Roper, T.J. \& Posadas-Andrews, A. (1981). Are schedule-induced drinking and displacement activities casually related? Quarterly Journal of Experimental Psychology, 33B, 181-193.

Sanger, D.J. (1986). Drug taking as adjunctive behavior. Em S.R. Goldberg \& LP. Stolerman (Orgs.), Behavioral analysis of drug dependence (pp. 123-160). New York: Academic Press.

Santarém, E.M.M. \& Silva, M.T.A. (1993). Efeito da clomipramina sobre a polidipsia induzida por esquema [Resumo]. Em Comissão de Pesquisa do Instituto de Psicologia da Universidade de São Paulo (Org.), Resumos de comunicações científicas, III 
Congresso Interno do Núcleo de Pesquisa em Neurociências e Comportamento (p. 60). São Paulo.

Segai, E.F. (1972). Induction and the provenance of operants. Em G.M. Gilbert \& J.R. Millenson (Orgs.), Reinforcement Behavioral Analysis (pp. 1-33). New York: Academic Press.

Segal, E.F., Oden, D.L. \& Deadwyler, S.A. (1965). Determinants of polydipsia: Free-reinforcement schedules. Psychonomic Science, 3, 11-12.

Shearon, T.O. \& Allen, J.D. (1989). Schedule-induced pellet pouching in the golden hammster. Bulletin of the Psychonomic Society, 27, 355-357.

Shurtleff, D., Delamater, A.R. \& Riley, A.L. (1983). A reevaluation of the CS hypothesis for schedule-induced polydipsia under intermittent schedules of pellet delivery. Animal Learning \& Behavior, 11,241-254.

Skinner, B.F. (1938). The behavior of organism. New York: AppletonCentury.

Staddon, J.E.R. (1977). Schedule-induced behavior. EmW.K. Honig \& J.E.R. Staddon (Orgs.), Handbook of operant behavior (pp. 125-152). Englewood Cliffs, N.J.: Prentice-Hall.

Staddon, J.E.R. \& Simmelhag, V.L. (1971). The "superstition" experiment: A reexamination of its implications for the principles of adaptative behavior. Psychological Review, 78, 3-43.

Staddon, J.E.R. \& Ayres, S.L. (1975). Sequential and temporal properties of behavior induced by a schedule of periodic food delivery. Behavior, 54, 26-49.

Tang, M. \& Falk, J.L. (1988). What schedule -induced polydipsia can tell us about alcoholism. Alcoholism: Clinical and Experimental Research, 12, 577-585.

Timberlake, W. (1982). Control and schedule-induced behavior. Animal Learning \& Behavior, 10, 535-536.

Timberlake, W. \& Lucas, G.A. (1985). The basis of superstitious behavior: Chance contingency, stimulus substitution, or appeti- tive behavior? Journal of the Experimental Analysis of Behavior, 44, 279-299.

Timberlake, W. \& Lucas, A.G. (1991). Periodic water, interwater, and adjunctive behavior in a 24-hour multiresponse environment. Animal Learning \& Behavior, 19, 369-380.

Todd, J.T., Cunningham, L.A., Janes, A.A., Mendelson, J., \& Morris, E.K. (1997). The generation and maintenance of scheduleinduced polydipsia in normal male rats without weight reduction. Physiology \& Behavior, 62, 1385-1390.

Wetherington, C.L. (1979). Schedule-induced drinking: Rate of food delivery and Herrnstein's equations. Journal of the Experimental Analysis of Behavior, 32, 323-333.

Wetherington, C.L. (1982). Is adjunctive behavior a third class of behavior? Neuroscience \& Biobehavioral Review, 6, 325-350.

Wetherington, C.L. \& Brownstein, A.J. (1977). Schedule control of eating by fixed-time schedules of water presentation. Animal Learning \& Behavior, 7, 38-40.

Wetherington, C.L. \& Brownstein, A.J. (1982). Comment on Roper's discussion of the language and generality of scheduleinduced behavior. Animal Learning \& Behavior, 10, 537-539.

Wetherington, C.L. \& Riley, A (1985). Differences in food consumption under intermittent and continuous reinforcement schedules of water delivery: Some implications for scheduleinduced behavior. Animal Learning \& Behavior, 13, 31-37.

Wilson, S. \& Spencer, W.B. (1975). Schedule-induced polydipsia: Species limitations. Psychological Reports, 36, 863-866.

Woods, A., Smith, C, Szeweza, K.M., Durn, R.W., Cornfeldt, M. \& Corbett, R. (1993). Selective serotonin re-uptake inhibitors decrease schedule-induced polydipsia in rats: A potencial model for obsessive compulsive disorder. Psychopharmacology, 112, 195-198.
Recebido em 05.05.1999

Primeira decisão editorial em 18.11.1999

Versão final em 07.01.2000

Aceito em 17.03.2000 